\title{
Prognostic modeling of the curvilinear graphene selective hydrogenation process for the formation of optical scheme components for nanophotonics
}

\author{
Hussein Safaa Mohammed Ridha ${ }^{1,2}$, S.I. Kharitonov ${ }^{1,3}$, V.S. Pavelyev ${ }^{1}$

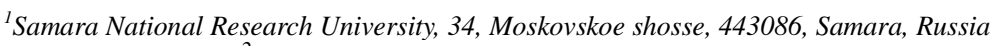 \\ ${ }^{2}$ University of Karbala, 56001, Karbala, Iraq \\ ${ }^{3}$ Image Processing Systems Institute - Branch of the Federal Scientific Research Centre "Crystallography and Photonics" of Russian Academy of Sciences, 151 \\ Molodogvardejskaya Street, 443001, Samara, Russia
}

\begin{abstract}
The article deals with modeling of curvilinear graphene hydrogenation process. During the hydrogen atoms addition, the maximum stresses shift from the region of the edge atoms to the central region of the structure. The ionization potential of curvilinear graphene begins to increase even with an insignificant hydrogen atom concentration on its surface. To vary the energy gap value of the curvilinear graphene spectrum, a high concentration of hydrogen atoms is necessary.
\end{abstract}

Keywords: graphene; graphane; nanocarbon structures; optical properties

\section{Introduction}

Nowadays, one of the promising directions in the field of nano- and bioelectronics is the development of new devices based on functionalized graphene. Today, the functionalization of graphene is one of the most effective ways to manage the properties of graphene material in order to expand the boundaries of its possible application in electronics and optics. The production of graphene nanostructures, functionalized with hydrogen, is an intensively developing direction of the modern nanoindustry, as well as the study of their properties. The hydrogen-functionalized graphene layer is a promising material for nanoelectronics and has received the name graphane in the literature. For the first time, graphane was experimentally obtained by the staff of the laboratory of Manchester University with the participation of Geim and Novoselov in 2009 by placing the graphene monolayer in hydrogen plasma. Graphane has two-dimensional, hexagonal crystalline structure. Hydrogen atoms are attached on both sides of the carbon atom plane by chemical bonds. According to the graphane sizes, graphite nanoparticles and nanobelts should be distinguished. The sizes of nanoparticles differ no more than in 3 times and do not exceed $100 \mathrm{~nm}$ in various directions.

The discovery of graphane have created the background for research its properties and searching for possible applications. In particular, graphane has unique optical properties. It has been established that the dielectric constant of graphane nanobelts does not depend on the shape of the belt edges and its width. In addition, as a result of studying the graphane optical properties, it has been shown that there is a moderate anisotropy with respect to the type of light polarization. The attention of researchers is also attracted to the study of the graphane thermal properties. For example, in [1] the authors investigate the graphane nanobelts thermal conductivity using the nonequilibrium Green's function method. In this paper it has been shown that the graphane thermal conductivity can be effectively controlled by the edge shape, the width, and also by the hydrogen vacancy concentration. In particular, the ballistic graphane nanobelts thermal conductivity usually increases with the belt width.

A promising area for graphane studies is the research of the graphane magnetic properties. It have been established in [2] that hydrogenated graphene demonstrates weak ferromagnetism in a wide range of temperatures down to room temperature, the nature of which is determined by the features of the graphane atomic structure itself.

As consequence of its unique physicochemical properties, graphane finds applications in a wide variety of scientific and technical fields. In particular, this material can be used in hydrogen economy. It has been found that heating of graphane leads to the atomic hydrogen release. Consequently, graphane can be considered as one of the most effective ways of storing hydrogen. Another important application of graphane will be its use in nanoelectronics as a basis for printed circuits with conductive and non-conductive areas on a sheet of graphane. One more possible application of graphane is the field of biosensorics. In the experimental work [3], the possibility of using graphane for electrochemical detection by applying graphan biomarkers is considered.

In one of the recent papers [4], the authors have studied theoretically the possibility of the existence of a two-dimensional doped graphane superconducting state. According to the results presented in the paper, doped graphane is a promising candidate for the creation of superconductors with a critical temperature higher than that of copper oxides. Another promising field of graphane application is its use as thermoelectric materials for thermionic devices. It is predicted that disordered armchair graphane nanobelts with low thermal conductivity can become a basis for creating thermoelectric materials.

\section{Methods of investigation}

In the work, the ionization potential and the energy gap, determined from the electronic spectrum, have been considered as the electron-energy characteristics of curvilinear graphene adsorbing hydrogen. The composite electron spectrum has been calculated with the close coupling method. Fig. 1 shows the energy levels diagram with an indication of the energy gap and the 
ionization potential. The ionization potential is determined by the last filled energy level (HOMO), and the energy gap is the interval between the last filled (HOMO) and the first vacant level (LUMO).

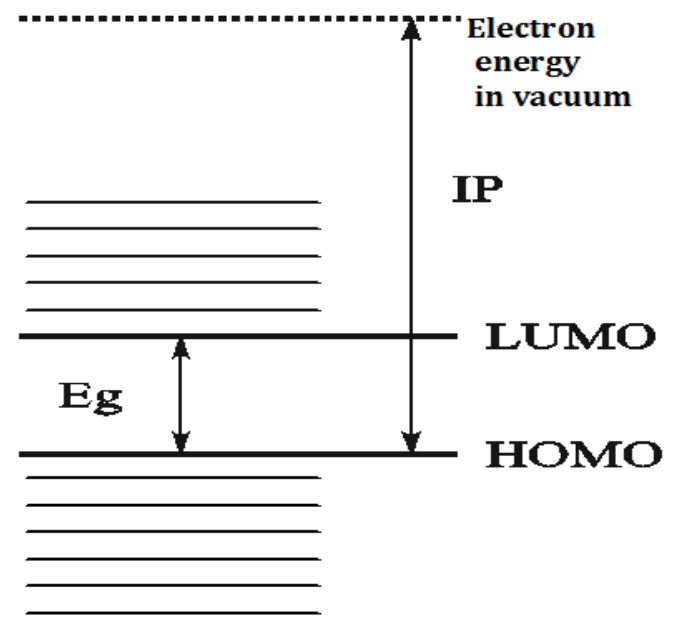

Fig. 1. An approximate diagram for the energy levels arrangement in the electronic spectrum with an indication of the HOMO and LUMO levels.

The change in the ionization potential and the energy gap of the electron spectrum during the process of curvilinear graphene selective hydrogenation is shown in the graphs represented by Fig. 2 and Fig. 3.

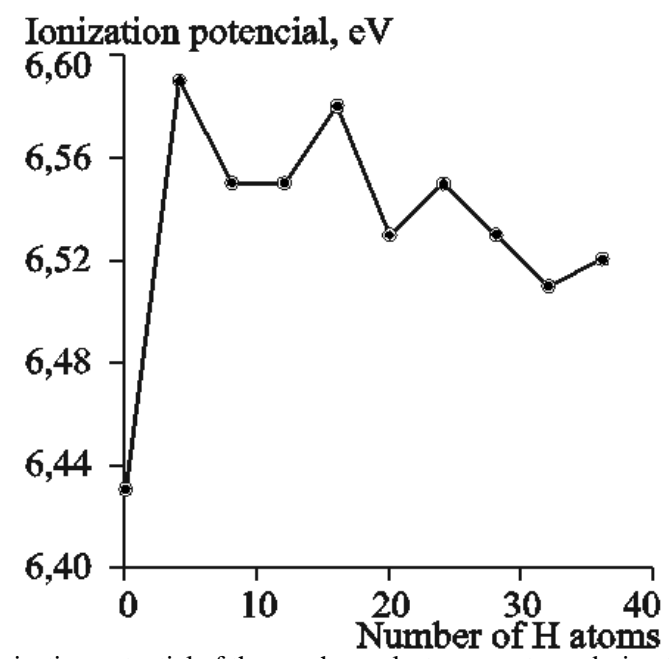

Fig. 2. The change in the ionization potential of the graphene electron spectrum during the hydrogen atoms addition.

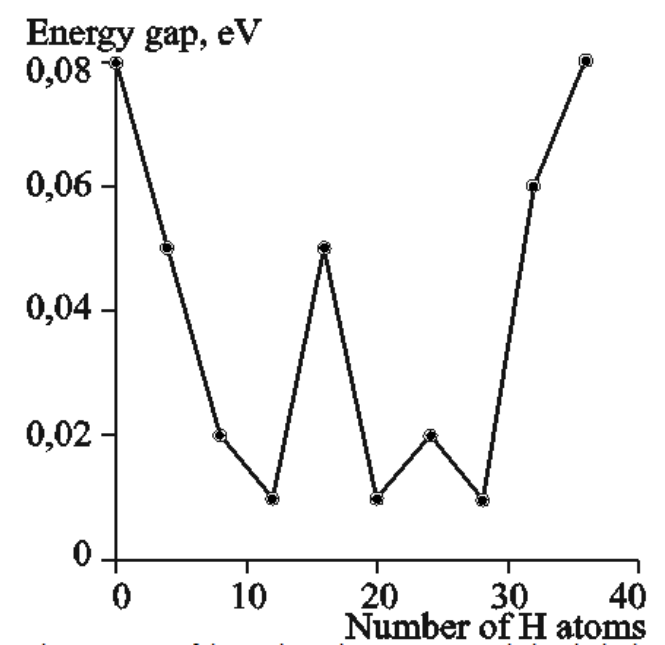

Fig. 3. The change in the energy gap of the graphene electron spectrum during the hydrogen atoms addition.

It can be seen from the graphs, even at the moment of addition of the first group of hydrogen atoms, the ionization potential of the structure increases steeply from 6.43 to $6.59 \mathrm{eV}$, and then changes in small limits near the value of $6.55 \mathrm{eV}$. Nevertheless, the obtained results indicate that the work function value, conclusions about which can be made from the ionization potential value, generally increases during the chemical adsorption of hydrogen by graphene, and hence the emissivity of such graphene structures decreases. The energy gap of the graphene electron spectrum, as seen from the graph in Fig. 3, varies discontinuously, 
linearly decreasing during the addition of the first groups of hydrogen atoms, and then changing in an alternating manner, increasing or decreasing. However, the range of values in which the gap varies evidence that the hydrogen atom concentration for the graphene fragment of given sizes considered in the work is insufficient to change the curvilinear graphene conductivity type from a semimetal to a semiconductor, or even to a dielectric.

Further, we have investigated the change in the density-of-states (DOS) distribution of curvilinear graphene with a gradually increasing of the hydrogen atom concentration. The energy spectrum of curvilinear graphene in which the energy of each molecular orbital was represented as a spectral line has been constructed in order to calculate DOS. The intensities of all the lines have been set to one. After that each line has been replaced by a Gaussian distribution with a half-width at a given halfheight of $0.1 \mathrm{eV}$. The intensities of all distributions for each energy value have been added up.

In constructing the partial electron density of atomic orbitals $\mathrm{x}$, the intensity of each line corresponding to the molecular orbital $\mathrm{y}$ has been assumed to be equal to the sum of the squared coefficients of the atomic orbitals $\mathrm{x}$ in the expansion of method of linear combinations of atomic orbitals (MO LCAO) of orbital y. Further, the algorithm for the partial density of states was analogous to the algorithm for constructing the total density of states.

The results of calculating the distribution of the $\pi$-electronic states density of the initial curvilinear graphene are shown in Fig. 4. The vertical line in the figure indicates the HOMO level. The figure shows that there are two characteristic symmetrical peaks of approximately equal intensity in the DOS distribution, one of which is in the valence band, the other is in the conduction band, and also a small cluster of electronic states near the HOMO level.

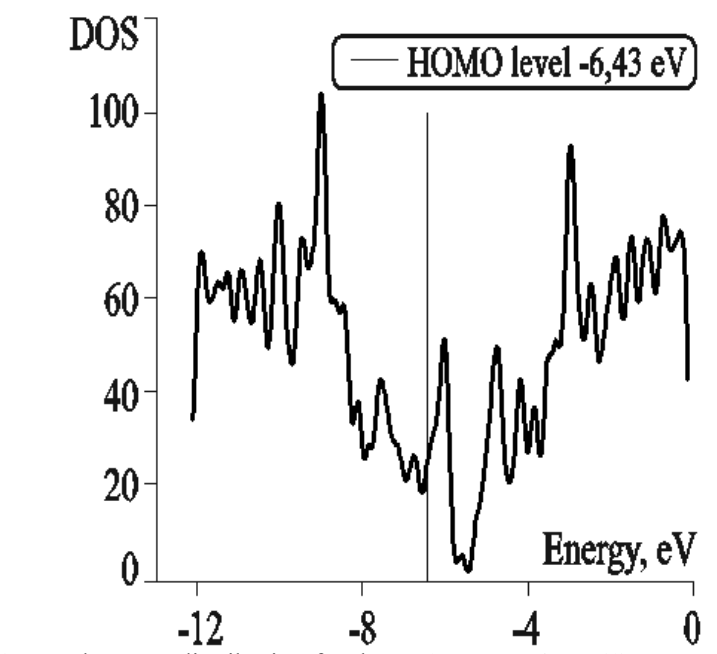

Fig. 4. The DOS distribution for the $\pi$-electrons of curvilinear graphene.

Further similar calculations and constructions were performed for curvilinear graphene with a different number of hydrogen atoms. The change in the DOS distribution for $\pi$-electrons of curvilinear graphene at each of the stages of hydrogen atom addition is shown in Fig. 5. It can be seen from the graph that the hydrogen addition causes a shift of the DOS characteristic towards the conduction band. The general character of the arrangement of peaks with maximum intensity also changes. The most significant changes are observed near the HOMO level and along the edges of the valence band as well as the conduction band. Near the HOMO level, the level density increases at each of the stages of hydrogen addition, while along the edges of the conduction and valence bands the density of states decreases in an oscillating manner.

\section{Main results}

The aim of this work is to determine the patterns of hydrogen atom chemical adsorption on curvilinear graphene using computer simulation methods.

In the course of studying the process of selective hydrogenation of curvilinear graphene, new physical patterns were revealed:

- From the energy point of view, chemical addition of hydrogen atoms to curvilinear graphene atoms with the greatest stress will be beneficial;

- During the addition of hydrogen atoms, the maximum stresses shift from the region of the edge atoms to the central region of the structure;

- The ionization potential of curvilinear graphene begins to increase even with an insignificant concentration of hydrogen atoms on its surface;

- To vary the energy gap value of the curvilinear graphene spectrum, a high concentration of hydrogen atoms is required;

The chemical addition of even a small number of hydrogen atoms leads to a shift in the DOS distribution toward the conduction band and the peak intensity redistribution near the HOMO level and along the band edges. 


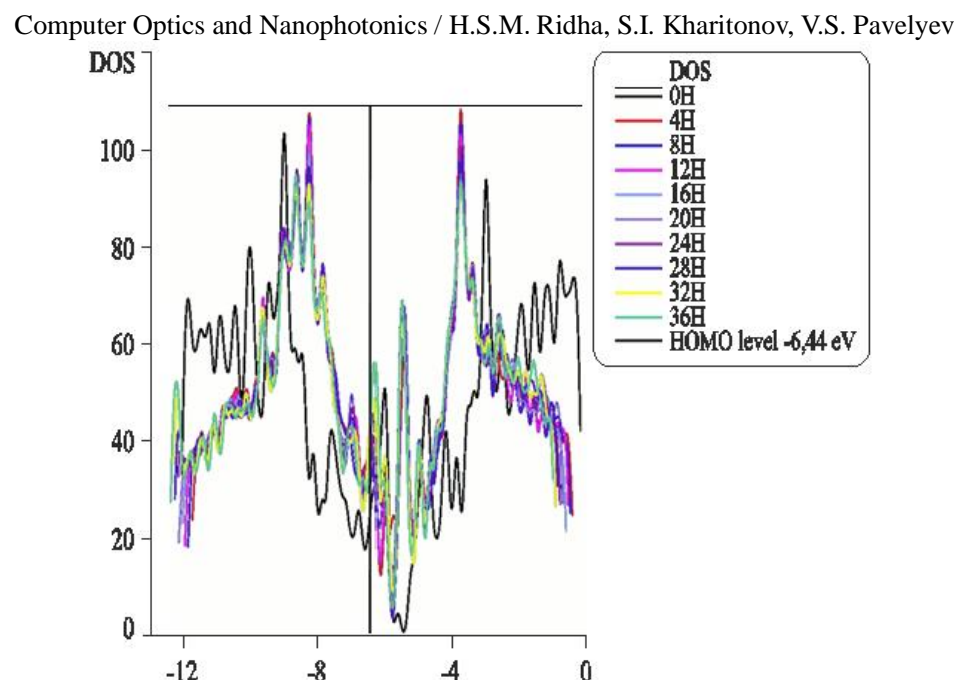

Fig. 5. Change in the DOS distribution of graphene during the addition of hydrogen atoms.

\section{Conclusion}

Thus, on the ground of the obtained results, an energetically advantageous mechanism of selective hydrogenation of curvilinear graphene has been proposed to control the charge carrier motion in the structure. The proposed mechanism can be used to form conductive areas in modern electronic circuits and to produce components of optical elements.

\section{References}

[1] Li D, Xu Y, Chen X, Li B, Duan W. Tunable anisotropic thermal conduction in graphane nanoribbons. Applied Physics Letters 2014; $104: 143108$.

[2] Eng AYS, Poh HL, Sanek F, Marysko M, Matejkova S, Sofer Z, Pumera M. Searching for Magnetism in Hydrogenated Graphene: Using Highly Hydrogenated Graphene Prepared via Birch Reduction of Graphite Oxides. ACS Nano 2013; 7: 5930-5939.

[3] Peng Q, Dearden AK, Crean J, Han L, Liu S, Wen X, De S. New materials graphyne, graphdiyne, graphone, and graphane: review of properties, synthesis, and application in nanotechnology. Nanotechnology, Science and Applications 2014; 7: 1-29.

[4] Durajski AP. Influence of hole doping on the superconducting state in graphane. Superconductor Science and Technology 2015; 28: 035002-8 pp. 